\title{
Self-paced Movement Intention Detection from Human Brain Signals: Invasive and Non-invasive EEG
}

\author{
Eileen Lew ${ }^{1}$, Ricardo Chavarriaga ${ }^{1}$, Huaijian Zhang ${ }^{1}$, Margitta Seeck ${ }^{2}$ and José del R. Millán ${ }^{1}$
}

\begin{abstract}
Neural signatures of humans' movement intention can be exploited by future neuroprosthesis. We propose a method for detecting self-paced upper limb movement intention from brain signals acquired with both invasive and noninvasive methods. In the first study with scalp electroencephalograph (EEG) signals from healthy controls, we report single trial detection of movement intention using movementrelated potentials (MRPs) in a frequency range between 0.1 to $1 \mathrm{~Hz}$. Movement intention can be detected above chance level $(p<0.05)$ on average $460 \mathrm{~ms}$ before the movement onset with low detection rate during the non-movement intention period. Using intracranial EEG (iEEG) from one epileptic subject, we detect movement intention as early as $1500 \mathrm{~ms}$ before movement onset with accuracy above $90 \%$ using electrodes implanted in the bilateral supplementary motor area (SMA). The coherent results obtained with non-invasive and invasive method and its generalization capabilities across different days of recording, strengthened the theory that self-paced movement intention can be detected before movement initiation for the advancement in robot-assisted neurorehabilitation.
\end{abstract}

\section{INTRODUCTION}

Decoding of voluntary movements before the action is highly important towards implementing practical brainmachine interface (BMI) technology for intuitive neuroprosthesis. Most experimental studies for BMI technology use cue-based protocols instead of voluntary (self-paced) actions that are stimulus-independent. These protocols pose further challenges in the understanding of human volition [1].

Recently, it was suggested that event-related desynchronization (ERD) in the alpha and beta EEG bands can be used for detecting finger movement onset prior to movement execution [2]. Besides ERD, slow cortical potentials (SCPs) $(<1 \mathrm{~Hz})$, known as movement-related potentials (MRPs), also contain early information about movement intention. In line with this, decoding of multi-directional upper limb movements using very low frequency EEG signals has also been reported [3]. The goal of this paper is to use the existing knowledge on MRPs [4], [5] to determine how early the movement intention can be detected in self-paced paradigms. To this end, this paper presents a unified decoding approach

*This work is supported by the European ICT Programme Project FP7224631 and SNSF Swiss National Center of Competence in Research (NCCR) Robotics.

${ }^{1}$ E. Lew, R. Chavarriaga, H. Zhang, and J. d. R. Millán are with Defitech Chair in Non-Invasive Brain-Machine Interface, Center for Neuroprosthetics, School of Engineering, Swiss Federal Institute of Technology Lausanne (EPFL), 1015 Lausanne, Switzerland. eileen. lew@a3.epfl.ch jose.millandepfl.ch

${ }^{2}$ M. Seeck is with Laboratory of Presurgical Epilepsy Evaluation, Program of Functional Neurology and Neurosurgery, University Hospital of Geneva, 1211 Geneva, Switzerland. tested on two experiments using non-invasive scalp EEG signals and intracranial EEG (iEEG).

\section{EXPERIMENTS}

\section{A. Non-invasive (EEG)}

Eight subjects (three female and seven right-handed subjects with average age of $29.33 \pm 2.06$ ) with no known history of neurological abnormalities or musculo-skeletical disorders participated in the experiment. The EEG and electromyogram (EMG) potentials were acquired with a portable ActiveTwo measurement system from BioSemi using 64 integrated electrodes arranged in the modified 10/20 International System at the sampling rate of $2048 \mathrm{~Hz}$.

The experimental workspace consisted of four targets buttons (up, down, left and right), and a home position button (center) on the horizontal plane as shown in Figure 1 (right). The recordings were conducted in a normal office environment to reflect reaching movement in a real-life scenario.

Subjects were instructed to perform natural self-paced center-out reaching tasks with their dominant arm. They were asked to fixate their eyes on a cross in the middle of the vertical plane as shown in Figure 1 (left) to ensure minimal eye movement-related artifacts in the recording. As shown in Figure 2, each trial began with the subject placing their dominant hand on the home position. After $500 \mathrm{~ms}$, an auditory cue informed the subject which target to reach. Subjects can initiate the movement whenever they wish, but at least $2 \mathrm{~s}$ after the presentation of the target cue. This constraint was imposed to avoid the stimulus-dependent activity. The movement onset was recorded as the time when the subject released the center button. Trials were discarded from analysis if the subject started the movement before $2 \mathrm{~s}$ or reached the wrong target. An average of 188 trials per all subjects were recorded with an average preparation time ( $\left.T_{\text {onset }}\right)$ is $5.03 \pm 1.77 \mathrm{~s}$.
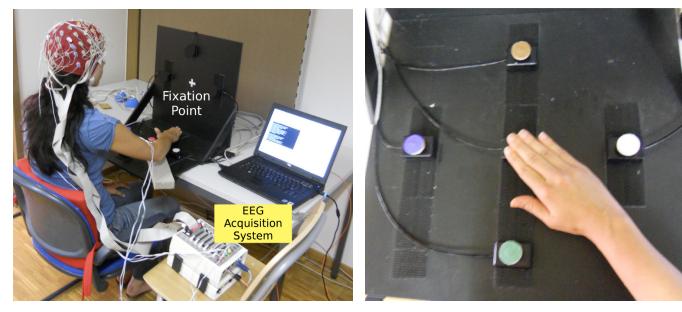

Fig. 1. Experimental setup (left) and top view of the targets position (right) 


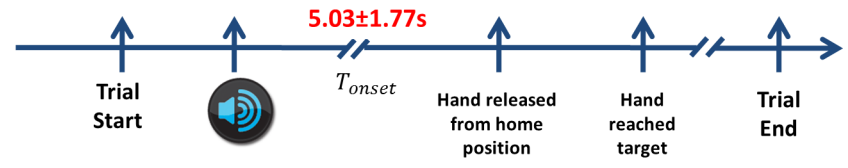

Fig. 2. The timeline of the experimental protocol with the average $T_{\text {onset }}$ across all subjects of $5.03 \pm 1.77 \mathrm{~s}$ for the EEG study.

\section{B. Invasive (iEEG)}

One epileptic patient participated in this recording following the usual clinical procedure at University Hospital of Geneva (HUG) [6]. The iEEG activity was recorded using 93 depth electrodes surgically implanted in the cerebral cortex of the brain (e.g., supplementary motor area (SMA), orbitofrontal (OF) cortex, hippocampal regions, etc.) using the Deltamed recording device. The reference electrode was located at scalp position $\mathrm{Cz}$ in the 10-20 International EEG System. The iEEG signals were sampled at $1024 \mathrm{~Hz}$ and filtered between $0.01 \mathrm{~Hz}$ and $1 \mathrm{KHz}$.

The subject was asked to fixate on a cross in the middle of a computer screen with four possible targets shown on the screen. The subject was asked to press a key to start the trial. After $500 \mathrm{~ms}$, an arrow appeared at the center of the screen, pointing to the intended target. As with the previous paradigm, subject was required to wait at least two seconds before starting the reaching movement where movement onset was the time when the subject released the key. The average onset time was $3.32 \pm 1.28 \mathrm{~s}$. The subject only performed a wrist movement rather than the entire arm reaching movement with her dominant arm (right). There were two days of recording from this subject, which were analyzed separately.

\section{METHODS}

Both the EEG and iEEG signals were downsampled to $256 \mathrm{~Hz}$ and filtered with a narrow band zero-phase noncausal IIR filter with cutoff frequencies of 0.1 and $1 \mathrm{~Hz}$. This frequency range has been reported to better capture anticipatory-related SCPs [7].
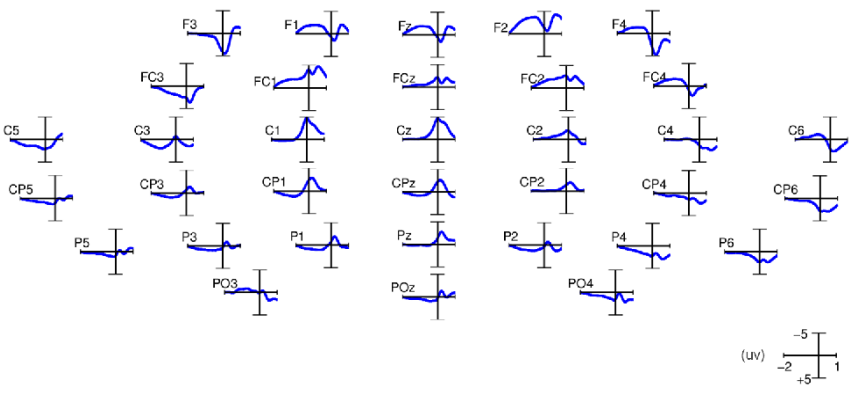

Time (ms)

Fig. 3. Grand averages MRPs filtered between 0.1 to $1 \mathrm{~Hz}$ for all the right-handed subjects $(n=7)$ participating in the EEG study.

In the EEG study, common average referencing (CAR) was used to remove the global background activity based on all the recorded channels [8]. However, the EEG channels (in particular the peripheral electrodes) could be contaminated by noise interference and muscular movements. We used regression analysis to assess the influence of electrooculograph (EOG) artifacts on each EEG channel [9]. Channels having high correlation with the EOG components were discarded from the montage before performing CAR, resulting in the use of 34 channels as depicted in Figure 3. Epochs were extracted from $2 \mathrm{~s}$ before the movement onset until $1 \mathrm{~s}$ after. For the purpose of classification, channels were manually selected based on grand average MRPs (shown in Figure 3 ). The channels chosen were $\mathrm{C} 1, \mathrm{Cz}, \mathrm{C} 2, \mathrm{CP} 1, \mathrm{CPz}, \mathrm{CP} 2$, in agreement with existing literature on MRPs [4], [5]. EMG signals were acquired bipolarly over the musculus biceps brachii and were processed to obtain envelopes of activity [10]. The purpose of this study is to verify that the detection of movement intention is not due to muscular artifacts. The onset of EMG activity was defined as the time when the average EMG activity exceeds a threshold value of $\mu+3 \sigma$, where $\mu, \sigma$ are the mean and standard deviation of EMG signals of a one-second window after the target cue [11].

For the iEEG study, we selected a total of 10 electrodes implanted in the SMA region. This region has been reported as the main cortical generator of MRPs [12], [13], [14]. Furthermore, a recent EEG study comparing contingent negative variation (CNV) potentials with MRPs (cue-based vs. selfinitiated action) showed significant role of the contralateral SMA region for self-initiated movements, as opposed to the dorsal premotor cortex (PMd) being more involved in externally cued preparatory processes of movement [15]. Similarly, another EEG study reported longer lasting activity in SMA during spontaneous tasks and in pre-motor area (PMA) during an instructed condition [16]. To assess the potential implication of other brain areas in this type of movements, we also performed the same analysis using 10 electrodes in the OF region. This region has been reported to be involved in planning and decision making [17].

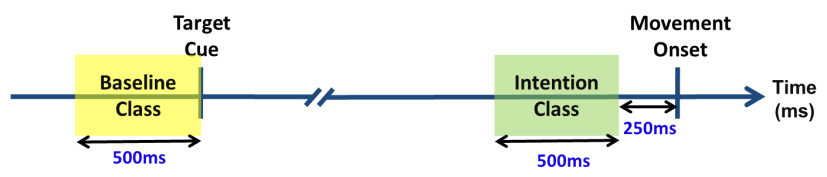

Fig. 4. Segments of data used for training the classifier.

In order to detect the movement intention from both EEG and iEEG signals, we categorized the signals into two parts: the baseline (idle) period and the movement intention (active) period as shown in Figure 4. During the idle period, $500 \mathrm{~ms}$ before the target cue, we assumed that there was no on-going movement preparation activity. The active period, defined at [-750 -250] $\mathrm{ms}$ before the movement onset, is expected to contain useful information for detecting movement intention. A linear discriminant analysis (LDA) classifier was trained using features computed by down-sampling the signal from $256 \mathrm{~Hz}$ to $8 \mathrm{~Hz}$ (4 data points per window for each selected channel) for both classes (idle vs. active). To test the performance of the classifier, we used a $500 \mathrm{~ms}$ sliding window shifted every $10 \mathrm{~ms}$. We reported the percentage 
of trials detected as movement intention (i.e. corresponding to the active class) at each time step. This percentage is expected to be very low at the moment of the auditory cue and gradually increase towards the movement onset. Due to the small number of training samples, we performed a 5-fold cross-validation to evaluate the performance of the proposed method. To find out how early the intention to move can be detected above chance level (95\% confidence interval using the Wilcoxon rank sum test), we obtained the first time when a group of 5 consecutive samples was recognized as movement intention. We calculated the actual chance level by random shuffling of training data labels, performed 10 times using 5-fold cross validation.

\section{RESULTS}

\section{A. Non-invasive EEG}

We report the time of movement intention detection, with respect to both the movement onset and EMG activity, and the maximum detection rate (MDR) in Table I where the negative value refers to time before movement onset.

TABLE I

MOVEMENT INTENTION DETECTION USING EEG SIGNALS.

\begin{tabular}{|c|c|c||c|c|}
\hline \multirow{2}{*}{ Subject ID } & \multicolumn{2}{|c||}{ Time Above Chance(ms) } & \multicolumn{2}{c|}{ Maximum Performance } \\
\cline { 2 - 5 } & Onset & EMG & MDR & Time $(\mathrm{ms})$ \\
\hline a5 & -500 & -281 & 0.85 & -110 \\
a8 & -500 & -289 & 0.70 & -240 \\
b5 & -290 & 14 & 0.83 & -150 \\
c2 & -400 & -130 & 0.84 & -100 \\
d6 & -570 & -292 & 0.72 & -120 \\
e7 & -510 & -194 & 0.80 & -140 \\
e8 & -460 & -179 & 0.71 & -190 \\
f1 & -450 & -223 & 0.69 & -290 \\
\hline \hline Average \pm std & $\mathbf{- 4 6 0} \pm \mathbf{8 5}$ & $\mathbf{- 1 9 7} \pm \mathbf{1 0 3}$ & $\mathbf{0 . 7 6} \pm \mathbf{0 . 0 7}$ & $\mathbf{- 1 6 7} \pm \mathbf{6 8}$ \\
\hline
\end{tabular}

On average, movement intention can be detected above chance level across subjects at $460 \mathrm{~ms}$ before movement onset and $197 \mathrm{~ms}$ before arm muscular activation. The average maximum detection rate was 0.76 , peaking at about $167 \mathrm{~ms}$ before movement onset. Except for subject b5, where the intent to move was detected $14 \mathrm{~ms}$ after EMG onset, EEGbased detection has achieved at least $130 \mathrm{~ms}$ before the EMG onset.

We also studied when movement intention can be detected from the EMG signal. To model the movement class, we take the window (between $-500 \mathrm{~ms}$ and $0 \mathrm{~ms}$ ) because the grand average EMG activity showed activity $250 \mathrm{~ms}$ before the movement onset.

TABLE II

MOVEMENT INTENTION DETECTION USING EMG SIGNAL.

\begin{tabular}{|c|c|c||c|c|}
\hline \multirow{2}{*}{ Subject ID } & \multicolumn{2}{c||}{ Time Above Chance(ms) } & \multicolumn{2}{c|}{ Maximum Performance } \\
\cline { 2 - 5 } & Onset & EMG & MDR & Time $(\mathrm{ms})$ \\
\hline a5 & -70 & 149 & 0.99 & 70 \\
a8 & -100 & 110 & 0.98 & 410 \\
b5 & -80 & 225 & 0.99 & 430 \\
c2 & -110 & 160 & 1.00 & 40 \\
d6 & 30 & 250 & 0.91 & 700 \\
e7 & -80 & 90 & 0.91 & 120 \\
e8 & -60 & 221 & 0.89 & 50 \\
f1 & -60 & 167 & 0.86 & 30 \\
\hline \hline Average \pm std & $\mathbf{- 6 6} \pm \mathbf{4 3}$ & $\mathbf{1 7 2} \pm \mathbf{5 7}$ & $\mathbf{0 . 9 4} \pm \mathbf{0 . 0 5}$ & $\mathbf{2 3 0} \pm \mathbf{2 5 0}$ \\
\hline
\end{tabular}

The results in Table II showed that movement can be detected from EMG activity at a time point close to the movement onset (average $66 \mathrm{~ms}$ before). Interestingly, the EMG classifier detects movement intention with higher rate, but later than the EEG classifier. We can thus conclude that detection of movement intention from EEG signals is not due to muscular artifacts and movement intention could be decoded from brain signals before muscular activation.

\section{B. Invasive $E E G$}

Figure 5 shows the average rate of movement intention detection, from time $2500 \mathrm{~ms}$ before movement onset to $1000 \mathrm{~ms}$ after, classified using contralateral SMA electrodes with data from Day 1. Movement intention detection exceeds chance level at $1900 \mathrm{~ms}$ before movement onset and has a peak value of 0.98 at $260 \mathrm{~ms}$ before movement onset. The result for Day 2 showed similar trends as Day 1, with movement intention detected at time $1400 \mathrm{~ms}$ before movement onset. The peak detection rate was 0.99 at $320 \mathrm{~ms}$ before movement onset (c.f. Table III).

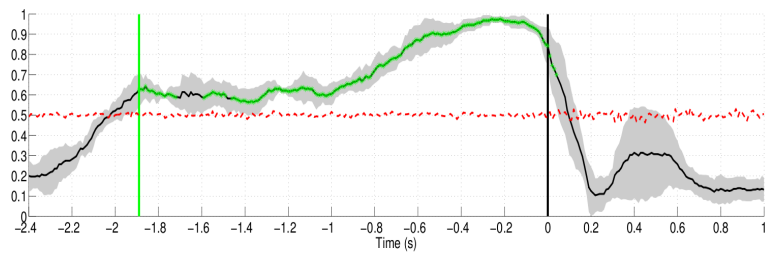

Fig. 5. Performance of Day 1 using electrodes implanted in the contralateral SMA. The green vertical line depicts the sample with detection rate significantly above chance level $(\mathrm{p}<0.05)$. Y-axis refers to the rate of movement intention detection.

We also investigated movement detection using signals from the ipsilateral SMA with results shown in Table III. In this case, the movement intention detected on Day 1 exceeds chance level at $1200 \mathrm{~ms}(700 \mathrm{~ms}$ later than contralateral SMA) and on Day 2, at $900 \mathrm{~ms}$ (500 ms later). The MDR was 0.96 at $440 \mathrm{~ms}$ before onset using data for Day 1 and 0.98 at $220 \mathrm{~ms}$ for Day 2. This showed movement intention related information in the ipsilateral SMA. It has been observed that a bilateral appearance of MRPs in the SMA, with MRPs more prominent on the contralateral side [13]. Similarly, we also observed smaller negativity on the ipsilateral channels from the grand averages of MRPs (c.f. Figure 3).

TABLE III

MOVEMENT INTENTION DETECTION FROM SMA REGIONS.

\begin{tabular}{|c|c|c|c|c|c|c|}
\hline \multirow[t]{2}{*}{ Day } & \multicolumn{3}{|c|}{ Contralateral SMA } & \multicolumn{3}{|c|}{ Ipsilateral SMA } \\
\hline & $\begin{array}{c}\text { Time } \\
\text { Above } \\
\text { Chance }\end{array}$ & MDR & $\begin{array}{l}\text { Time } \\
\text { MDR }\end{array}$ & $\begin{array}{c}\text { Time } \\
\text { Above } \\
\text { Chance }\end{array}$ & MDR & $\begin{array}{l}\text { Time } \\
\text { MDR }\end{array}$ \\
\hline 1 & -1900 & 0.98 & -260 & -1200 & 0.96 & -440 \\
\hline 2 & -1400 & 0.99 & -320 & -900 & 0.98 & -220 \\
\hline
\end{tabular}

When using orbital frontal electrodes for classification, we observed lower performance than classification with SMA electrodes, with performance shown in Table IV. The results showed that the frontal region does contain information about movement intention, although less discriminant than SMA. 
TABLE IV

MOVEMENT INTENTION DETECTION FROM FRONTAL REGIONS.

\begin{tabular}{|c|c|c|c|c|c|c|}
\hline \multirow[t]{2}{*}{ Day } & \multicolumn{3}{|c|}{ Contralateral OF } & \multicolumn{3}{|c|}{ Ipsilateral OF } \\
\hline & $\begin{array}{c}\text { Time } \\
\text { Above } \\
\text { Chance }\end{array}$ & MDR & $\begin{array}{l}\text { Time } \\
\text { MDR }\end{array}$ & $\begin{array}{c}\text { Time } \\
\text { Above } \\
\text { Chance }\end{array}$ & MDR & $\begin{array}{l}\text { Time } \\
\text { MDR }\end{array}$ \\
\hline 1 & -1030 & 0.78 & -90 & -760 & 0.78 & -130 \\
\hline 2 & -1370 & 0.88 & -110 & -1030 & 0.85 & -80 \\
\hline
\end{tabular}

To assess the generalization capabilities of this method, we performed the classification of data from Day 2 using a decoder trained on Day 1. Figure 6 shows the rate of movement intention detection using electrodes from the contralateral and ipsilateral SMA. The results show good generalization using the same features from Day 1 to Day 2 with MDR at 0.95 at $360 \mathrm{~ms}$ before movement onset using the contralateral SMA electrodes and 0.93 at $350 \mathrm{~ms}$ before movement onset with the ipsilateral SMA.
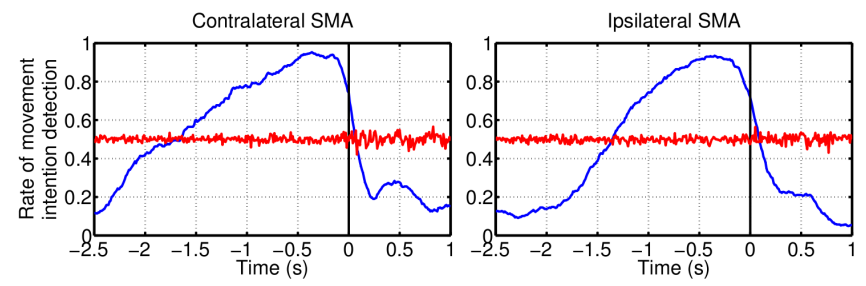

Fig. 6. Rate of movement intention detection of Day 2, tested with classifier trained with data from Day 1 using contralateral and ipsilateral SMA electrodes. The red line shows the chance level.

\section{DISCUSSION AND CONCLUSIONS}

We have shown that self-paced movement intention can be detected based on MRPs computed in a narrow frequency range between 0.1 to $1 \mathrm{~Hz}$ around $500 \mathrm{~ms}$ before movement onset. Our results are consistent with the time characteristics of average MRPs [4], [5]. The approach yields high sensitivity -i.e., high detection rates close to the movement onset and low detection rates during non-movement intention period.

Similar trend of performance is observed from intracranial electrodes implanted in the bilateral SMA region as early as $1500 \mathrm{~ms}$ before the movement onset. Our results are in line with studies on single unit monkey recordings showing that the SMA contain neurons that become active up to $2000 \mathrm{~ms}$ before the initiation of self-paced movements [18]. Furthermore, we have shown the generalization capability of using the SMA electrodes as an important feature for detecting movement intention from one day to another for the design of neuroprosthesis. Better performances obtained using iEEG signals could be due to a higher signal-to-noise ratio [19] than scalp EEG recordings. We observed an earlier detection of movement intention in the invasive EEG study, which could be due to a more sustained planning related activity within the SMA and OF area [16], [14].

The results reported are encouraging and can be further extended for its practical application in designing neuroprosthesis by testing the proposed method in an online implementation with both able-bodied and disabled users.

\section{ACKNOWLEDGMENT}

Authors warmly thank G. Garipelli, M. Tavella, and A. Biasiucci for their help with recordings and discussions.

\section{REFERENCES}

[1] P. Haggard, "Human volition: towards a neuroscience of will," Nat Rev Neurosci, vol. 9, no. 12, pp. 934-946, 2008.

[2] O. Bai, V. Rathi, P. Lin, D. Huang, H. Battapady, D.-Y. Fei, L. Schneider, E. Houdayer, X. Chen, and M. Hallett, "Prediction of human voluntary movement before it occurs," Clin Neurophysiol, vol. 122, no. 2, pp. $364-372,2011$.

[3] T. J. Bradberry, R. J. Gentili, and J. L. Contreras-Vidal, "Reconstructing three-dimensional hand movements from noninvasive electroencephalographic signals." J Neurosci, vol. 30, no. 9, pp. 3432-3437, Mar 2010.

[4] H. Kornhuber and L. Deecke, "Changes in the brain potential in voluntary movements and passive movements in man: Readiness potentials and reafferent potentials." Pflugers Arch Gesamte Physiol Menschen Tiere, vol. 284, pp. 1-17, 1965.

[5] B. Libet, E. W. Jr., and C. Gleason, "Readiness-potentials preceding unrestricted spontaneous vs. pre-planned voluntary acts," Electroen Clin Neuro, vol. 54, no. 3, pp. 322 - 335, 1982.

[6] G. Pourtois, L. Spinelli, M. Seeck, and P. Vuilleumier, "Temporal precedence of emotion over attention modulations in the lateral amygdala: Intracranial ERP evidence from a patient with temporal lobe epilepsy." Cogn Affect Behav Neurosci, vol. 10, no. 1, pp. 83-93, Mar 2010.

[7] G. Garipelli, R. Chavarriaga, and J. d. R. Millán, "Single trial recognition of anticipatory slow cortical potentials: The role of spatiospectral filtering," in Proceedings of the 5th International Conference on Neural Engineering, 2011.

[8] O. Bertrand, F. Perrin, and J. Pernier, "A theoretical justification of the average reference in topographic evoked potential studies," Electroen Clin Neuro, vol. 62, no. 6, pp. 462-464, 1985.

[9] A. Schlögl, C. Keinrath, D. Zimmermann, R. Scherer, R. Leeb, and G. Pfurtscheller, "A fully automated correction method of EOG artifacts in EEG recordings." Clin Neurophysiol, vol. 118, no. 1, pp. 98-104, Jan 2007.

[10] V. C. K. Cheung, L. Piron, M. Agostini, S. Silvoni, A. Turolla, and E. Bizzi, "Stability of muscle synergies for voluntary actions after cortical stroke in humans." PNAS, vol. 106, no. 46, pp. 19563-19568, 2009

[11] Abbink, V. D. Bilt, and V. D. Glas, "Detection of onset and termination of muscle activity in surface electromyograms," J Oral Rehabil, vol. 25, no. 5, pp. 365-369, 1998.

[12] A. Ikeda, H. O. Lüders, H. Shibasaki, T. F. Collura, R. C. Burgess, H. H. Morris, and T. Hamano, "Movement-related potentials associated with bilateral simultaneous and unilateral movements recorded from human supplementary motor area." Electroen Clin Neuro, vol. 95, no. 5, pp. 323-334, 1995.

[13] A. Ikeda, H. O. Lüders, R. C. Burgess, and H. Shibasaki, "Movementrelated potentials recorded from supplementary motor area and primary motor area," Brain, vol. 115, no. 4, pp. 1017-1043, 1992.

[14] I. Rektor, "Scalp-recorded Bereitschaftspotential is the result of the activity of cortical and subcortical generators - a hypothesis," Clin Neurophysiol, vol. 113, no. 12, pp. 1998 - 2005, 2002.

[15] M.-K. Lu, N. Arai, C.-H. Tsai, and U. Ziemann, "Movement related cortical potentials of cued versus self-initiated movements: Double dissociated modulation by dorsal premotor cortex versus supplementary motor area rTMS," Hum Brain Mapp, vol. 33, no. 4, pp. 824-839, 2012.

[16] G. Thut, C.-A. Hauert, P. Viviani, S. Morand, L. Spinelli, O. Blanke, T. Landis, and C. Michel, "Internally driven vs. externally cued movement selection: a study on the timing of brain activity," Cognitive Brain Res, vol. 9, no. 3, pp. $261-269,2000$.

[17] J. D. Wallis, "Orbitofrontal cortex and its contribution to decisionmaking." Аnпи Rev Neurosci, vol. 30, pp. 31-56, 2007.

[18] K. Okano and J. Tanji, "Neuronal activities in the primate motor fields of the agranular frontal cortex preceding visually triggered and selfpaced movement." Exp Brain Res, vol. 66, no. 1, pp. 155-166, 1987.

[19] G. Buzsáki, C. A. Anastassiou, and C. Koch, "The origin of extracellular fields and currents - EEG, ECoG, LFP and spikes." Nat Rev Neurosci, vol. 13, no. 6, pp. 407-420, 2012. 